\title{
Effects of tDCS over the right DLPFC on attentional disengagement from positive and negative faces: An eye-tracking study
}

\author{
Alvaro Sanchez $^{1}$ (D) $\cdot$ Marie-Anne Vanderhasselt ${ }^{1} \cdot$ Chris Baeken $^{1} \cdot$ Rudi De Raedt $^{1}$
}

Published online: 5 August 2016

(C) Psychonomic Society, Inc. 2016

\begin{abstract}
The aim of this study was to increase insight in the neural substrates of attention processes involved in emotion regulation. The effects of right dorsolateral prefrontal cortex (i.e., DLPFC) stimulation on attentional processing of emotional information were evaluated. A novel attention task allowing a straightforward measurement of attentional engagement toward, and attentional disengagement away from emotional faces was used. A sample of healthy participants received 20 minutes of active and sham anodal transcranial direct current stimulation (i.e., tDCS) applied over the right DLPFC on 2 separate days and completed the attention task after receiving real or sham stimulation. Compared to sham stimulation, tDCS over the right DLPFC led to impairments in attentional disengagement from both positive and negative faces. Findings demonstrate a causal role of right DLPFC activity in the generation of attentional impairments that are implicated in emotional disturbances such as depression and anxiety.
\end{abstract}

Keywords Selective attention - Attentional control ·

Emotion $\cdot$ DLPFC $\cdot$ Neurostimulation

Selective attention is a cognitive process that serves to filter relevant external and internal information to be processed and can be initiated either in automatic or controlled ways (Corbetta \& Schulman, 2002). In the context of emotional processing, selective attention is a crucial mechanism that influences our emotional experience and functioning (Gross,

Alvaro Sanchez

alvaro.sanchezlopez@ugent.be

1 Department of Experimental Clinical and Health Psychology, Ghent University, Henri Dunantlaan 2, B-9000 Ghent, Belgium
Sheppes, \& Urry, 2011; Koole, 2009) by determining how the organism perceives and interprets emotional information in the environment (Everaert, Duyck, \& Koster, 2014; Sanchez, Everaert, De Putter, Mueller, \& Koster, 2015). Individual differences in attentional processing of emotional information have been found to have a primary involvement in emotion regulation processes (Gross et al., 2011; Joormann \& D'Avanzato, 2010). Whereas healthy individuals under transient induced negative mood states direct their attention more frequently to positive stimuli (Sanchez, Vazquez, Gomez, \& Joormann, 2014) and are faster in shifting attention away from negative stimuli (Ellenbogen, Schwartzman, Stewart, \& Walker, 2002), individuals suffering from anxiety and depression are characterized, among other biases, by sustained attentional processing of negative information (Armstrong \& Olatunji, 2012). Individual differences in these attention patterns are associated with recovery from transient negative mood states (Ellenbogen et al., 2002; Sanchez, Vazquez, Marker, LeMoult, \& Joormann, 2013; Sanchez et al., 2014).

Neuroimaging studies demonstrate an interactive network of corticolimbic pathways playing a central role in the topdown regulation of emotions (Johnstone, van Reekum, Urry, Kalin, \& Davidson, 2007; Ochsner \& Gross, 2005; Seminowicz et al., 2004; Wager, Davidson, Hughes, Lindquist, \& Ochsner, 2008). Specifically, a functional balance between ventral (ventral anterior cingulate cortex [ACC]; limbic structures) with dorsal compartments in the brain (dorsal ACC, dorsolateral prefrontal cortex [DLPFC]) is necessary for maintaining homeostatic emotional control (Ochsner \& Gross, 2005). These brain structures are also involved in attentional processing of emotional information (Fragopanagos, Kockelkoren, \& Taylor, 2005; Liotti \& Mayberg, 2001), with the DLPFC as an important region for the implementation of top-down attentional control (MacDonald, Cohen, Stenger, \& 
Carter, 2000; Vanderhasselt, De Raedt, Baeken, Leyman, \& D'Haenen, 2006). Therefore, emotional attention biases associated to the dysregulation of emotional states can be understood as the result of failures in top-down attentional control implemented by the DLPFC (De Raedt, Vanderhasselt, \& Baeken, 2015).

It has been consistently observed that impairments in the ability to disengage attention from negative information that characterizes emotional disorders are commonly related to dysfunctional DLPFC activity (De Raedt \& Koster, 2010). For instance, attention biases toward threat are characterized by right hemispheric hyperactivation of regions including frontal (orbital, prefrontal, anterior cingulate), parietal, and subcortical regions, such as the amygdala (for reviews, see Disner, Beevers, Haigh, \& Beck, 2011; Nitschke \& Heller, 2005). Recent research has examined the causal role of the right DLPFC in emotional attention processes by employing experimental neurostimulation techniques to manipulate right DLPFC activity, such as repetitive transcranial magnetic stimulation (rTMS) or transcranial direct current stimulation (tDCS). These procedures consist of noninvasive techniques that cause transient changes in activity of particular cortical areas that last beyond the stimulation period (Kluger \& Triggs, 2007; Nitsche et al., 2008). rTMS is an increasingly used neurostimulation technique where electrical stimulation is delivered by an electromagnetic coil placed above the scalp in which a high-intensity current is rapidly turned on and off. The resulting time-varying magnetic field generated passes to the surface of the brain area, where it induces weak electric currents to flow in the underlying neurons. tDCS is a relatively new but promising technique of neuromodulation to manipulate the membrane potential of neurons. tDCS modulates spontaneous cortical activity through delivery of a constant low-intensity direct current (e.g., 0.5-2 mA) via an anodal electrode and a cathodal electrode attached to the scalp surface, inducing intracerebral current flows. Research has demonstrated an increase in cortical excitability due to anodal stimulation (Nitsche \& Paulus, 2000, 2001), whereas cathodal stimulation decreases cortical activity (Nitsche \& Paulus, 2000; Nitsche et al., 2003).

Leyman, De Raedt, Vanderhasselt, and Baeken (2009) examined the transient effects of high-frequency (HF) rTMS applied over the right DLPFC - which increases cortical excitability —on the attentional processing of emotional information (happy and sad faces) of healthy volunteers. In line with the existence of the aforementioned neurocircuitry of emotion regulation, results supported an involvement of the right DLPFC in attentional processing, evidenced by an instant impairment of the attentional inhibition of sad faces following HF-rTMS. Furthermore, De Raedt et al. (2010) replicated and extended this finding in a study where healthy individuals completed an exogenous cueing task involving attentional processing of angry faces in the fMRI scanner following
HF-rTMS of both right and left DLPFC. Results showed that right-sided neurostimulation resulted in impaired attentional disengagement from angry faces, whereas left-sided stimulation resulted in decreased attentional engagement toward angry faces. It is important that impaired disengagement was associated with decreased activation within the right DLPFC, dorsal ACC, and left superior parietal gyrus, combined with increased activity within the right amygdala during the task performance. Specifically, results from De Raedt et al. (2010) show that such impairments are based on reduced activation of right DLPFC during disengagement trials (as measured by event-related fMRI) as a result of neurostimulation, which creates hemispheric imbalances as observed in emotional disorders (i.e., higher right, lower left activation; Disner et al., 2011; Nitschke \& Heller, 2005). These findings provide important insights on the neural substrates of emotional attention processes implicated in emotional regulation (i.e., ability to inhibit or disengage attention from negative information).

However, further research is required given two limitations of the aforementioned studies: (1) the absence of direct measures of attention behavior (De Raedt et al., 2010; Leyman et al., 2009), and (2) the absence of positive stimuli (De Raedt et al., 2010), to test whether the observed effects are specific for negative stimuli or reflect general impairments in the inhibition of emotional information. First, the attention tasks employed by the aforementioned studies rely on manual reaction time measures as proxy estimations of attention allocation. For instance, in the spatial cueing task employed by De Raedt et al. (2010), longer reaction times to indicate the position of a target that appears at the opposite position of the screen (relative to where a negative cue was previously presented) are assumed to indicate a delayed attentional disengagement from negative information. However, in the absence of a continuous measurement of overt visual attention, it is difficult to disentangle the influence of emotional processes on attentional responses from other confounding factors, such as deficits in motor response or response selection biases. In addition, the absence of continuous measurement of overt visual attention may undermine the ability to effectively capture shifts in attention over time. The measurement of distinct attention components (i.e., attentional engagement vs. attentional disengagement; Posner \& Cohen, 1984) involved in emotional processing requires methods to differentiate these components in a more straightforward way, to test the hypothesis that the right DLPFC plays a role in specific attentional processes that require attentional control recruitment (i.e., attentional disengagement). In this study, a sample of healthy undergraduates completed a novel engagementdisengagement attention task based on eye tracking (Sanchez et al., 2013), after receiving a session of both 
active anodal tDCS and sham (placebo) stimulation over the right DLPFC. This design was aimed to establish the specific effects of right DLPFC stimulation over direct measures of engagement and disengagement components of emotional attention, including both negative and positive stimuli. According to predictions from neurocognitive models (Davidson 1992), and in line with the aforementioned studies using neurostimulation, higher right DLPFC activity induced by anodal tDCS should be specifically involved in disruptions of attentional control of negative information - namely, in the ability to inhibit or disengage attention away from intrusive negative information.

Second, although previous evidence indicates that dysfunctional right DLPFC activity may be specifically linked to impairments in the ability to disengage attention from negative information, the role of right DLPFC activity in attentional disengagement from positive information remains unclear. Traditional neurocognitive models argue that preferential processing of positive (approach related) and negative emotions (withdrawal related) would be lateralized towards the left and right prefrontal cortex, respectively (Davidson, Ekman, Saron, Senulis, \& Friesen, 1990). However, recent studies on lateralized effects of neurostimulation over the left DLPFC have shown general effects in implementing attentional control for all emotional information rather than specific effects for negative information. Nitsche et al. (2012) found that anodal tDCS compared to sham stimulation over left DLPFC had temporal effects in improving attentional detection of both disgusted and happy faces. These findings speak against the traditional view on valence lateralization. A recent study of healthy individuals tested the effectiveness of an experimental procedure to modify the attentional inhibition of different emotional stimuli in combination with left DLPFC stimulation (Clarke, Browning, Hammond, Notebaert, \& MacLeod, 2014). Results showed that participants receiving anodal tDCS in comparison to sham stimulation showed increases in the specific trained attentional inhibition pattern, irrespectively of its valence. Taken together, whereas recent evidence on the role of left DLPFC stimulation points toward general attentional control improvements, previous findings (De Raedt et al., 2010; Leyman et al., 2009) indicate that right DLPFC stimulation leads to attentional disengagement impairments for negative information. However, whether stimulation of the right DLPFC is related to general impairments in attentional control outcomes (i.e., attentional disengagement for both negative and positive information) remains unclear.

Only one study has tested this hypothesis (Leyman et al., 2009), reporting effects of right DLPFC stimulation on impaired inhibition of negative but not of positive facial stimuli. Nonetheless, further research is required to further illuminate this research question. The task employed by Leyman and colleagues (negative affective priming task [NAP]; Joormann, 2004) might limit the generalizability of the results. Specifically, the NAP task serves as an indicator of general inhibitory function that may be linked to a broader component of cognitive control (Joormann, Yoon, \& Zetsche, 2007). Moreover, a decrease in general attentional control for non-emotional stimuli has been previously reported as the result of rTMS over right DLPFC in a study testing the reorienting of attention during the performance of a cued visual choice task (Rounis et al., 2006).

In this study, the question of whether right DLPFC stimulation leads to negative-specific or general impairments in attentional disengagement from emotional information was tested by using direct measures of attentional engagement and disengagement processes through the analyses of the time it takes to move gaze toward and away from both positive and negative distractor faces. Based on previous findings (De Raedt et al., 2010; Leyman et al., 2009), we predicted that the stimulation of the right DLPFC, in comparison to sham stimulation of that area, would lead to the generation of specific impairments in emotional attentional processes recruiting attentional control (i.e., attentional disengagement). Furthermore, we explored whether such effect would reflect valence-specific (i.e., negative facial expressions; Leyman et al., 2009) or general attentional control impairments (i.e., both negative and positive facial expressions; Rounis, 2006).

\section{Method}

\section{Participants}

A sample of 30 undergraduates of Ghent University, with a mean age of 23.27 years $(S D=4.15)$ was recruited via Internet posting to participate in this study. All participants (10 males and 20 females) were right-handed, had normal or correctedto-normal (using glasses or contact lenses) vision, were not pregnant at the time of stimulation, and had no metal in or around their scalp. None reported a history of, or currently had, a neurological or psychiatric disorder (using the Dutch version of the MINI screen; Overbeek, Schruers, \& Griez, 1999; Sheehan et al., 1998). Moreover, participants were excluded if they reported a history of serious head injury or were having eye problems or difficulties in vision that were not corrected by the use of glasses or contact lenses. Participants gave their written informed consent and received 40 euro for their participation. The study was conducted in adherence to the Declaration of Helsinki and approved by the medical 
ethical committee of the University Hospital (2014/0433). Participants were recruited in the context of a larger project investigating neurocognitive markers of attentional deployment and cognitive control for emotional material. ${ }^{1}$

\section{Questionnaires}

Depressive symptoms Participants completed the Beck Depression Inventory-II (BDI-II; Beck, Steer, \& Brown, 1996; Van der Does, 2002), a 21-item self-report measure to assess depression severity. Respondents report on 4-point scales how much they have experienced depression symptoms over the last 2 weeks. This measure has shown excellent reliability and validity (Beck et al., 1996). In this study, internal consistency was good $(\alpha=.90)$ as well as test-retest reliability among sessions $(\mathrm{ICC}=.83, p=.001)$.

Anxiety symptoms Participants filled out the State-Trait Anxiety Inventory (STAI; Spielberger, Gorsuch, Lushene, Vagg, \& Jacobs, 1983; Van der Ploeg, Defares, \& Spielberger, 1980), a 20-item self-report measure of trait anxiety. All items are rated on a 4-point scale. Scores range from 20 to 80 , with higher scores correlating with greater anxiety. Internal consistency and test-retest reliability among sessions were $\operatorname{good}(\alpha=.91 ; \mathrm{ICC}=.91, p=.001$, respectively) in this study.

Mood state To evaluate temporary changes in mood before (Tpre) versus after (Tpost) receiving real and sham tDCS, mood ratings were administered using six Visual Analogue Scales (VAS) providing measures of fatigue, tension, anger, vigor, sadness, and cheerfulness (McCormack et al., 1988). Participants were asked to describe how they felt "at that moment" by indicating on horizontal $100-\mathrm{cm}$ lines whether they experienced the five aforementioned mood states, from totally not to very much.

\section{Transcranial direct current stimulation (tDCS)}

Direct electrical current was applied by a saline-soaked pair of surface sponge electrodes $\left(35 \mathrm{~cm}^{2}\right)$ and delivered by a batterydriven stimulator (DC-Stimulator Plus, neuroConn $\mathrm{GmbH}$ ). To stimulate the right DLPFC, the anode electrode was vertically positioned over $\mathrm{F} 4$ according to the 10-20 international

\footnotetext{
${ }^{1}$ Further cognitive control tasks in the project included (1) an emotional appraisal paradigm, consisting of viewing pleasant and unpleasant pictures, preceded by cues anticipating the emotional content of the pictures (previously employed by Vanderhasselt, Remue, Ng, \& De Raedt, 2014), and (2) a cued emotion control task (CECT) to measure proactive and reactive control for emotional information (previously employed by Vanderhasselt, De Raedt, et al., 2013). Both tasks where applied after completing the attentional engagement-disengagement task in both sessions.
}

system for electroencephalogram electrode placement. The cathode was horizontally placed over the contra lateral supraorbital area. This reference electrode location has been shown to be functionally ineffective in experimental designs (Nitsche et al., 2003). This electrodes placement and method of DLPFC localization is in accordance with prior tDCS studies (e.g., Giglia et al., 2014; Heinze et al. 2014) and has demonstrated significant effects on cognitive information processing in previous research (Vanderhasselt, De Raedt, et al., 2013; Vanderhasselt, Brunoni, Loeys, Boggio, \& De Raedt, 2013). A constant, direct current of $2 \mathrm{~mA}$, with $15 \mathrm{~s}$ of a ramp up and down, was applied for $20 \mathrm{~min}$. Excitability changes on cortical function using this technique and stimulation parameters are expected to last more than 1 hour (Nitsche et al., 2008). For sham stimulation (e.g., placebo stimulation condition), the electrodes were positioned similar to when administering real tDCS stimulation; however, the current was ramped down after 15 seconds. This procedure is a reliable sham condition because subjects are not able to discriminate real from sham stimulation (Nitsche et al., 2008).

\section{Attentional engagement-disengagement task}

Stimuli Stimuli consisted of pairs of pictures comprising an emotional and a neutral facial expression of the same person. Faces were selected from the Radboud Faces database (RaFD; Langner et al., 2010). Stimulus selection was based on data from a previous validation study of the RaFD (Langner et al., 2010), which measured the emotional discreteness of faces for the corresponding emotion and their valence and intensity ratings, among other parameters. Based on these data, 24 happy, disgusted, and sad expressions (12 men and 12 women for each emotional category), together with the corresponding neutral expression of the same actors, were selected as the stimuli for the current study. ${ }^{2}$ Two different types of negative emotional faces were included to evaluate with tDCS the findings from previous rTMS studies that have shown effects of right DLPFC stimulation in the generation of attentional disengagement impairments for both sad-related (i.e., sad faces; Leyman et al., 2009) and threat-related (i.e., angry faces; De

\footnotetext{
${ }^{2}$ The following RaDF frontal view models were selected for each of the three emotions plus their neutral pair: Rafd090_02_Caucasian_female, Rafd090_03_Caucasian_male, Rafd090_04_Caucasian_female, Rafd090_05_Caucasian_male, Rafd090_12_Caucasian_female, Rafd090 16 Caucasian female, Rafd090 18 Caucasian female, Rafd090 20 Caucasian male, Rafd090 21 Caucasian male, Rafd090_24_Caucasian_male, Rafd090_28_Caucasian_male, Rafd090 31 Caucasian female, Rafd090 32 Caucasian female, Rafd090_33_Caucasian_male, Rafd090_36_Caucasian_male, Rafd090 37 Caucasian female, Rafd090 38 Caucasian male, Rafd090 46 Caucasian male, Rafd090 47 Caucasian male, Rafd090_49_Caucasian_male, Rafd090_56_Caucasian_female, Rafd090 $57^{-}$Caucasian female, Rafd090 58 Caucasian female, Rafd090_61_Caucasian_female.
} 
Raedt et al., 2010) negative stimuli. Disgust instead of angry faces were used because current research points to a greater threat perception for disgust faces (e.g., Amir et al., 2005). Moreover, a previous study testing effects of tDCS over left DLPFC have demonstrated improvements in the attentional detection of both happy and disgust faces (Nitsche et al., 2012). Therefore, this set was considered ideal to test whether tDCS over right DLPFC leads to valence-specific or general attentional disengagement impairments.

In terms of emotional discreteness, according to Langner et al.'s (2010) validation data, the selected models showed a high percentage of discreteness for the proper emotions $(M=$ $88.83, S D=12.23)$. In terms of valence, disgusted and sad faces $(M=1.97, S D=.17$, and $M=2.04, S D=.20$, respectively) did not show significant differences $(p=.67)$, whereas happy faces $(M=4.29, S D=.24)$ significantly differed from both $(p=.001$, in both cases).

Experimental design The attention task comprised 72 trials (24 happy, 24 disgusted and 24 sad expressions paired with the corresponding neutral expression of the same actor), which were randomly presented for each participant. Emotional and neutral expressions were presented equally often on the left as on the right. The task also included six practice trials, followed by a brief pause before starting the actual trials. In total, the attention task lasted approximately 9 minutes.

Stimuli were displayed on a 22-inch Mitsubishi 2070SB color monitor using a Cambridge Research Systems ViSaGe visual stimulus generator. The stimulus generator had a contrast resolution of 14 bits per gun. The average luminance of the monitor was $104 \mathrm{~cd} / \mathrm{m}^{2}$ (4002 Td) and the output of the display was gamma corrected. The size of each face was $7.5 \mathrm{~cm}$ (width) $\times 11 \mathrm{~cm}$ (height). Pictures were centered on the screen, $39 \mathrm{~cm}$ apart (measured from their centers). Participants were seated approximately $75 \mathrm{~cm}$ from the screen's center, resulting in a visual angle of approximately 5.7 degrees between each picture's center and the screen's center.

The experimental design is presented in Fig. 1. Each trial started with the presentation of a black screen for $500 \mathrm{~ms}$, followed by the display of a white fixation cross

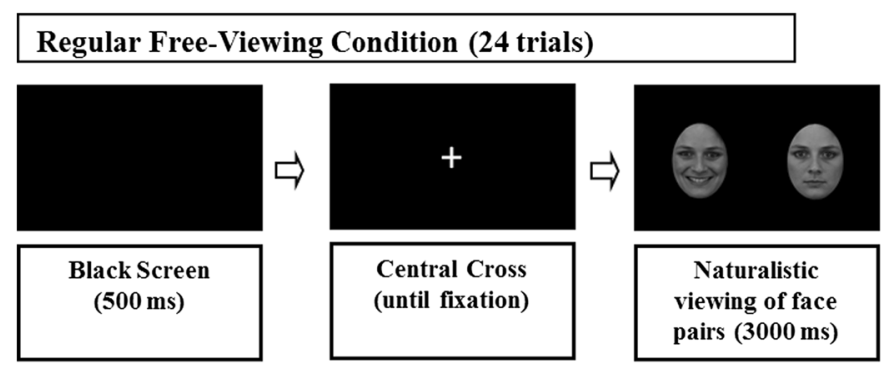

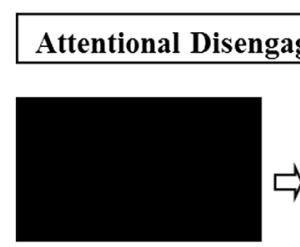
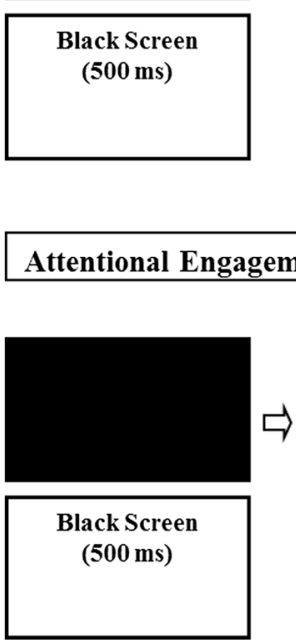
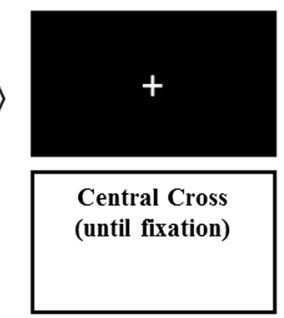

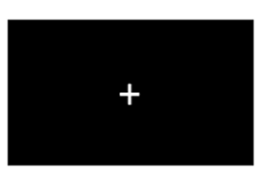

Central Cross (until fixation)

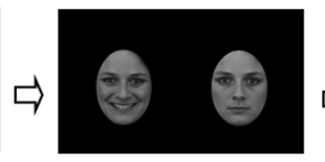

Naturalistic viewing of face pairs $(3000 \mathrm{~ms})$

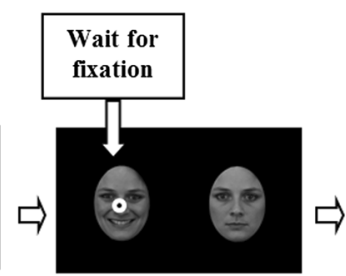

Wait for Fixation on the emotional face (minimum time: $100 \mathrm{~ms})$

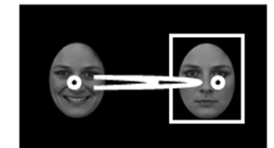

Response to the type of frame (until participant's response)

Fig. 1 Schematic of trial presentations in the attentional engagement-disengagement task 
in the middle of the screen. Immediately after the system detected a visual fixation of at least $200 \mathrm{~ms}$ in the cross area, a pair of faces (either happy-neutral, disgustedneutral or sad-neutral) was presented for 3,000 ms. An engagement-disengagement task similar to the one used by Sanchez et al. (2013) was performed after the 3,000 ms of natural processing stimulus presentation. This task allows the direct assessment of attentional engagement and disengagement patterns (Sanchez et al., 2013). The task comprised three conditions: (1) One third of the trials in each emotion condition (happy, disgusted, sad) assessed attentional engagement with emotional expressions. As shown in Fig. 1, this condition involved that after the $3,000 \mathrm{~ms}$ of naturally viewing, stimulus presentation did not continue until participants fixated on the neutral face for $100 \mathrm{~ms}$. When this fixation was detected, a frame consisting of a square or a circle appeared surrounding the opposite face (i.e., emotional face). Participants were instructed to direct their gaze toward that frame as quickly as possible and press one of two response keys on the keyboard to indicate the type of frame (i.e., square or circle). (2) Another third of the trials assessed disengagement from emotional expressions in each emotion condition. The procedure was similar, but, in this case, after the $3,000 \mathrm{~ms}$ of naturally viewing, stimulus presentation did not continue until participants fixated on the emotional face for $100 \mathrm{~ms}$ and then the frame appeared surrounding the opposite neutral face. (3) A last third of the trials included a regular free-viewing task for each emotion condition, in which after the $3,000 \mathrm{~ms}$ naturally viewing period, a new fixation cross appeared, indicating the start of the next trial.

Trials of engagement, disengagement, and regular freeviewing conditions for each emotional condition (i.e., happy-neutral, disgusted-neutral, sad-neutral) were randomly presented for each participant. Both types of frames were equally likely to appear in the left and right positions in all conditions. Criteria for identifying a first shift in gaze to the stimuli surrounded by the frame on each trial were as follows: (a) Participants were fixated on the opposite stimulus before the frame appeared, (b) eye movements occurred at least $100 \mathrm{~ms}$ after the frame appeared, (c) gaze was directed to the stimulus surrounded by a frame rather than remaining at the opposite position or being directed to a different area on the screen, and (d) participants made a fixation of at least $100 \mathrm{~ms}$ to the stimulus surrounded by a frame after shifting their gaze to it. Therefore, trials with visual fixations in the framed stimulus occurring before $200 \mathrm{~ms}$ (i.e., $100 \mathrm{~ms}$ of visual fixation on the opposite stimulus before the frame appearance plus at least $100 \mathrm{~ms}$ after the frame appearance) were excluded because they would not reflect eye movements in response to the task demand. Similarly, trials where initial visual fixations after the frame appearance did not occur in the framed stimulus were also excluded. Analyses were conducted on $83 \%$ of the data.

Eye tracker Participants' eye movements were recorded using a Cambridge Research Systems High-Speed Video Eyetracker (CRS HS-VET) with a spatial resolution of $0.05^{\circ}$ and accuracy of $0.125^{\circ}$ to $0.25^{\circ}$. This system employs a dualPurkinje eye-tracking method (see Crane \& Steele, 1985) and samples eye-gaze coordinates at $250 \mathrm{~Hz}$ (e.g., a coordinates' estimation every $4 \mathrm{~ms})$. The CRT monitor and the eye tracker were controlled using the CRS MATLAB toolboxes, with the eye-tracking system automatically synchronized to the stimulus presentation at the beginning of each trial. Eye position samples were converted to visual fixation data by using CRS MATLAB toolboxes. Visual fixations were defined as a minimum duration of $100 \mathrm{~ms}$ and a maximum fixation radius of 1 degree.

Attention indices Because our study aimed to test whether right DLPFC stimulation leads to negative-specific or general impairments in attentional disengagement from (as opposed to attentional engagement toward) emotional information, we focused our analyses on the direct measures of attentional engagement and disengagement processes. Therefore, the engagement-disengagement conditions employed after the $3,000 \mathrm{~ms}$ free viewing period served to establish direct measures of (1) attentional engagement (i.e., latency of the first shift in gaze from the neutral face to the emotional face surrounded by the frame in the engagement condition) and (2) attentional disengagement (i.e., latency of the first shift in gaze from the emotional face to the neutral face surrounded by the frame in the disengagement condition).

\section{Procedure}

Written informed consent was obtained from all participants. First, participants filled in the questionnaires, followed by the neuromodulation session. A single-blind randomized crossover within-subjects design was used, for which each participant received both real and sham (placebo) stimulation on two separated days. The order of both stimulation sessions (real tDCS and sham stimulation) was counterbalanced for each participant, with an interval of at least 48 hours (most participants had an interval of at least 1 week between real-sham stimulation sessions). All participants were exposed to a similar stimulation context during both stimulation sessions by asking them to remain seated and relaxed until the stimulation was finished. During both sessions, immediately after the end of the stimulation, participants performed the attentional engagement-disengagement paradigm during which eye movements were recorded. In addition, subjective mood ratings were recorded using the VASs at two time points in each 
session: at baseline, before receiving simulation, and approximately 60 minutes after stimulation, after completing the attentional engagement-disengagement task.

\section{Results}

\section{Participants' characteristics}

Demographic and self-reported characteristics of the sample are presented in Table 1. Participants were characterized by low depression and anxiety levels, as assessed by the screening measures, reducing potential confounding effects of these psychological dimensions in emotional attention processes in the study. Regarding depression levels, participants reported a low mean depressive symptom severity level of $5.14(S D=$ 7.54), with no participants reaching subclinical depression severity levels according to cut-off criteria (all BDI-II scores $<14$; see Beck et al., 1996). Regarding anxiety levels, participants reported mid to low anxiety trait levels $(M=42.4, S D=$ 11.28; STAI-T range: $20-80$ ).

\section{TDCS effects on mood}

Mean scores for each VAS score at both pre and post stimulation assessment (for both stimulation conditions, active and sham) are presented in Table 1.

Table 1 Emotional characteristics in each tDCS condition

\begin{tabular}{lrrrrr}
\hline Variables & \multicolumn{2}{c}{ Sham tDCS $(N=30)$} & & \multicolumn{2}{c}{ Active tDCS $(N=30)$} \\
\cline { 2 - 3 } \cline { 5 - 6 } & $M$ & $S D$ & & $M$ & \multicolumn{1}{l}{$S D$} \\
\hline BDI-II Depression & 4.91 & 7.42 & & 5.37 & 7.16 \\
STAI-T Trait Anxiety & 42.44 & 11.01 & & 42.76 & 12.44 \\
VAS Fatigue pre-tDCS & 2.88 & 2.05 & & 2.98 & 1.89 \\
VAS Fatigue post-tDCS & 5.36 & 2.04 & & 5.83 & 2.54 \\
VAS Vigor pre-tDCS & 5.72 & 2.11 & & 5.57 & 2.11 \\
VAS Vigor post-tDCS & 4.67 & 2.26 & & 4.08 & 2.32 \\
VAS Anger pre-tDCS & 0.87 & 1.20 & & 0.57 & 0.71 \\
VAS Anger post-tDCS & 2.23 & 2.51 & & 1.33 & 2.13 \\
VAS Tension pre-tDCS & 2.00 & 2.16 & & 1.25 & 1.55 \\
VAS Tension post-tDCS & 2.83 & 2.64 & & 2.35 & 2.69 \\
VAS Sadness pre-tDCS & 1.04 & 1.49 & & 0.97 & 1.28 \\
VAS Sadness post-tDCS & 1.18 & 1.53 & & 1.00 & 1.43 \\
VAS Cheerful pre-tDCS & 6.20 & 2.27 & & 6.28 & 2.05 \\
VAS Cheerful post-tDCS & 5.01 & 2.05 & & 5.13 & 2.10 \\
\hline
\end{tabular}

Note. $M=$ mean, $S D=$ standard deviation, $\mathrm{tDCS}=$ transcranial direct current stimulation, BDI-II = Beck Depression Inventory-II; STAI-T = State-Trait Anxiety Inventory-Trait subscale; VAS = Visual Analogue Scale
To examine effects of tDCS stimulation in mood changes, a $2 \times 2$ repeated-measures ANOVA was conducted, with stimulation (active vs. sham tDCS) and time (prestimulation, poststimulation) as within-subjects factors for each VAS (i.e., fatigue, tension, anger, vigor, sadness, and cheerful). Significant time effects were found for all VASs, fatigue: $F(1,28)=$ $63.54, p=.001, \eta^{2}=.69$; tension: $F(1,28)=6.07, p=.02$, $\eta^{2}=.18$; anger: $F(1,28)=9.37, p=.01, \eta^{2}=.25$; vigor: $F(1$, $28)=15.01, p=.001, \eta^{2}=.35$; cheerful: $F(1,28)=22.97, p$ $=.001, \eta^{2}=.45$, with the exception of sadness: $F(1,28)=$ $1.07, p=.31, \eta^{2}=.04$. Those effects were qualified by a general mood decrease across time, with fatigue, tension, and anger mood increases and vigor and cheerful mood decreases from prestimulation to poststimulation assessment. However, none of these main effects were qualified by significant stimulation by time interactions, fatigue: $F(1,28)=0.94$, $p=.34, \eta^{2}=.03$; tension: $F(1,28)=0.51, p=.48, \eta^{2}=.02$; anger: $F(1,28)=1.03, p=.32, \eta^{2}=.04$; vigor: $F(1,28)=$ $0.58, p=.45, \eta^{2}=.02$; sadness: $F(1,28)=0.11, p=.74, \eta^{2}=$ .01 ; cheerful: $F(1,28)=0.01, p=.99, \eta^{2}=.01$. Therefore, a single session of anodal tDCS over right DLPFC did not differently modify mood in comparison to sham stimulation, in line with previous research (see Remue, Baeken, \& De Raedt, 2016).

\section{TDCS effects on attentional engagement-disengagement}

Means and standard deviations for each attention index in each stimulation condition are reported in Table 2. To test differences among stimulation conditions in attentional engagement toward and disengagement from emotional information, a $2 \times 2 \times 3$ repeated-measures ANOVA was conducted, with stimulation (active vs. sham tDCS), attention component (disengagement vs. engagement) and emotion (happy faces vs. disgusted faces vs. sad faces) as within-subjects factors. First, analyses showed a significant interaction of attention component by emotion, $F(2,58)=7.76, p=.001, \eta^{2}=$ .21. Bonferroni-corrected comparisons showed that, in general, participants were characterized by significantly longer times to disengage attention from happy faces than from sad faces, $F(2,28)=3.83, p=.03, \eta^{2}=.21$, as well as by shorter times to engage attention toward happy faces than toward sad faces, $F(2,28)=6.82, p=.01, \eta^{2}=.33$.

Second, analyses revealed a significant interaction of stimulation by attention component, $F(1,29)=15.04, p=.001, \eta^{2}$ $=.34$. Bonferroni-corrected comparisons showed a general effect of tDCS on attentional disengagement from emotional faces, irrespective of their valence: compared to sham stimulation, tDCS over the right DLPFC generated longer times to disengage attention from emotional faces, $F(1,29)=12.91, p$ $=.01, \eta^{2}=.31$. In contrast, Bonferroni comparisons did not reveal significant differences between sham and tDCS stimulation in the time to engage attention toward emotional faces, 
Table 2 Attention indices in each tDCS condition

\begin{tabular}{|c|c|c|c|c|}
\hline \multirow[t]{2}{*}{ Attention index (in ms) } & \multicolumn{2}{|c|}{ Sham tDCS $(N=30)$} & \multicolumn{2}{|c|}{ Active tDCS $(N=30)$} \\
\hline & $M$ & $S D$ & $M$ & $S D$ \\
\hline Disengagement from happy faces & 348.92 & 63.26 & 379.16 & 71.72 \\
\hline Engagement toward happy faces & 345.22 & 66.86 & 339.91 & 58.22 \\
\hline Disengagement from disgusted faces & 329.47 & 63.13 & 369.68 & 56.19 \\
\hline Engagement toward disgusted faces & 364.60 & 87.64 & 328.98 & 85.57 \\
\hline Disengagement from sad faces & 318.30 & 50.39 & 342.76 & 73.01 \\
\hline Engagement toward sad faces & 374.79 & 80.27 & 368.01 & 57.77 \\
\hline
\end{tabular}

Note. $M=$ mean, $S D=$ standard deviation, $\mathrm{ms}=$ milliseconds, $\mathrm{tDCS}=$ transcranial direct current stimulation

$F(1,29)=1.26, p=.27, \eta^{2}=.04$. This general tDCS effect is shown in Fig. 2. Supporting a general impairment hypothesis, there was no significant three-way stimulation by attention component by emotion interaction, $F(2,58)=1.24, p=.29$, $\eta^{2}=.04$.

\section{Discussion}

The aim of this study was to increase our insight in the neural substrates involved in cognitive processes of emotional regulation. The DLPFC, among other brain regions, has been proposed as one of the key hubs linking attention and emotion regulation processes via the implementation of top-down control (e.g., MacDonald et al., 2000). Previous research has shown that transient neurostimulation of the right DLPFC in healthy individuals generates impairments in the ability to inhibit or disengage attention from negative information (De Raedt et al., 2010; Leyman et al., 2009), in the same way that

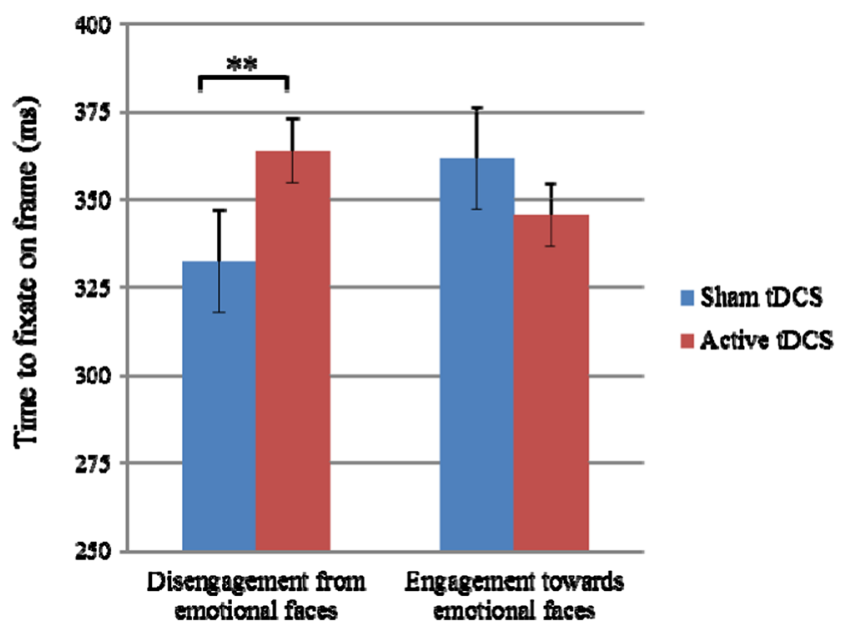

Fig. 2 Mean times of attentional disengagement from and attentional engagement toward emotional faces comparing sham and real tDCS conditions. Note $. \mathrm{ms}=$ milliseconds, $\mathrm{tDCS}=$ transcranial direct current stimulation. Error bars indicate standard error. $* * p<.01$ is commonly observed in individuals suffering from disorders characterized by emotional dysregulation (i.e., anxiety, depression; see Armstrong \& Olatunji, 2012). In this study, we aimed to replicate and extend these findings by employing a paradigm that allowed obtaining direct measures of gaze behavior involved in both attentional engagement toward and attentional disengagement from both negative and positive information. This innovative paradigm allowed us to disentangle separate components of emotional attention processing to clarify (1) the specific role of right DLPFC activity in the inhibition or disengagement of attention from emotional faces, in contrast to processes of attentional engagement towards them, and (2) whether such an attention disengagement impairment reflects a valence-specific impairment for negative faces, or whether it reflects a more general impairment in emotional attentional control (i.e., both negative and positive faces).

Whereas previous studies have shown effects of the stimulation of right DLPFC in proxy measures of attention (De Raedt et al., 2010; Leyman et al., 2009), this is the first study showing effects of right DLPFC stimulation in direct measures of gaze behavior. First, our results confirmed a specific effect after anodal tDCS of the right DLPFC on the attentional disengagement component. In comparison to sham stimulation, active tDCS over the right DLPFC significantly delayed attentional disengagement from emotional faces but had no effects on attentional engagement towards them. These effects were independent from mood state differences because tDCS had no differential effects on causing mood state changes before completing the attention task, which is in line with former neurostimulation research (Baeken, Leyman, De Raedt, Vanderhasselt, \& D'Haenen, 2006, 2008; see also Remue et al., 2016). Moreover, we tested the effects of tDCS in a healthy sample reporting low levels in subclinical dimensions of depression and anxiety, thereby reducing potential confounds due to these variables. Therefore, our results reflect impairments in attentional disengagement processes as a result of the direct action of anodal tDCS over the right DLPFC. This deficient attentional control is congruent with previous 
findings using different techniques of right DLPFC neurostimulation (i.e., HF-rTMS: De Raedt et al., 2010; Leyman et al., 2009) and points toward a role of this brain area in emotional attentional control. In contrast, initial detection or attentional engagement toward emotional information is proposed to be dependent of initial activity in limbic areas such as the amygdala (e.g., Morris et al., 1998), and consistently, no effects of DLPFC neurostimulation were observed in the attentional engagement component.

Second, our results indicate an involvement of the right DLPFC in general emotional attentional control rather than valence-specific effects in the processing of negative information. Consistent with previous findings, our results showed that attentional control impairments are observed in the attentional disengagement from different types of negative faces, including sad (Leyman et al., 2009) and threatening (De Raedt et al., 2010) faces. However, this effect in attentional control impairments was also observed in the attentional disengagement from positive information (i.e., happy faces). These results, although apparently contradictory with predictions from traditional neurocognitive models (e.g., Davidson, 1992), are congruent with recent findings pointing to a hemispheric lateralization of processes related to all emotional information rather than emotion-specific processes. Whereas previous studies using anodal tDCS over the left DLPFC have shown that such stimulation has effects on implementing attentional control for different emotional stimuli, irrespective of their valence (Clarke et al., 2014; Nitsche et al., 2012), our study is the first in demonstrating a general attentional control impairment for both positive (happy) and negative (disgust and sad) stimuli as a result of right DLPFC anodal stimulation. Furthermore, our results are also in line with previous research suggesting a direct action of anodal tDCS over the right DLPFC in the generation of deficient general attentional control (i.e., nonemotional cues; Rounis et al., 2006).

This study should be noted for its methodological rigor. The novel eye-tracking paradigm employed in the study allowed for the direct assessment of engagement and disengagement components of emotional attention processing in controlled conditions, an aspect not addressed by previous neurostimulation research (De Raedt et al., 2010; Leyman et al., 2009). Our results provide support for the notion that right DLPFC alterations may be involved in the generation of impairments in attentional disengagement from emotional information, a commonly observed problem in emotional disorders that is linked to sustained negative affect and emotional dysregulation (e.g., Sanchez et al., 2013). Specifically, attentional disengagement impairments observed in emotional disorders (De Raedt \& Koster, 2010) may be lateralized to the right DLPFC, which would be related to hemispheric imbalances in this area, as commonly reported by previous research (e.g., Blackhart, Minnix, \& Kline, 2006; Debener et al., 2000; Gotlib, 1998).

Some limitations should be acknowledged. The effects induced by anodal tDCS are not limited to the DLPFC region, but reflect the involvement of a network including the DLPFC. Neuroimaging data has demonstrated that tDCS over the DLPFC influences other cortical and subcortical sites beyond the targeted region, modulating, for instance, resting-state functional connectivity in distinct functional brain networks (e.g., Keeser et al., 2011; Stagg et al., 2013). Anodal tDCS might stimulate not only the proposed region and underlying neural network but also adjacent cortical areas, resulting in the modulation of more remote areas lying between the two tDCS electrodes. Future studies should consider applying neuroimaging to investigate the neural connectivity changes during the attentional engagement with and attentional disengagement from emotional stimuli following tDCS. Further research would also benefit from including measurements of adverse effects associated with tDCS (see Brunoni et al., 2011) to control for potential side-effects of neurostimulation (e.g., discomfort, headache).

Our findings, if replicated and extended with the combination of neuroimaging techniques (see De Raedt et al., 2010), provide support for the rationale of new therapies for emotional disorders based on the use of neuromodulation techniques. These approaches aim to balance the activation of right and left DLPFC areas by inhibiting the right DLPFC or activating the left DLPFC in combination with attentional control training (De Raedt et al., 2015). It has already been observed that the addition of tDCS of the left DLPFC to multiple sessions of cognitive control training can be promising to increase long-term effects (Brunoni et al., 2011; Segrave, Arnold, Hoy, \& Fitzgerald, 2013). Although further research is necessary to replicate and extend these findings, current evidence points out to the need of integrating cognitive interventions aimed to generate improvements in attentional control of emotional information together with neuromodulation techniques directed to balance the activation of DLPFC areas involved in such process (for a rationale, see De Raedt et al., 2015). In this sense, initial research testing the use of DLPFC neuromodulation in combination with attentional control training is starting to show that this may be a promising intervention tool to improve attentional impairments in emotional disorders (Heeren, Baeken, Vanderhasselt, Philippot, \& De Raedt, 2015; Heeren, De Raedt, Koster, \& Philippot, 2013). Furthermore, current evidence also opens the possibility of developing new diagnostic tests based on biological markers, by identifying target brain areas involved in target maladaptive cognitive processes. 
Acknowledgements and Funding Information This research was supported by a grant of the Research Foundation Flanders (FWO), awarded to Alvaro Sanchez, and a grant (BOF16/GOA/017) for a Concerted Research Action of Ghent University, awarded to Rudi De Raedt. This work was also supported by the Ghent University Multidisciplinary Research Partnership, "The Integrative Neuroscience of Behavioral Control."

\section{References}

Amir, N., Klumpp, H., Elias, J., Bedwell, J. S., Yanasak, N., \& Miller, L. S. (2005). Increased activation of the anterior cingulate cortex during processing of disgust faces in individuals with social phobia. Biological Psychiatry, 57(9), 975-981. doi:10.1016/j. biopsych.2005.01.044

Armstrong, T., \& Olatunji, B. O. (2012). Eye tracking of attention in the affective disorders: A meta-analytic review and synthesis. Clinical Psychology Review, 32(8), 704-723. doi:10.1016/j.cpr.2012.09.004

Baeken, C., Leyman, L., De Raedt, R., Vanderhasselt, M. A., \& D'haenen, H. (2006). Lack of impact of repetitive high frequency transcranial magnetic stimulation on mood in healthy female subjects. Journal of Affective Disorders, 90(1), 63-66. doi:10.1016/j. jad.2005.09.013

Baeken, C., Leyman, L., De Raedt, R., Vanderhasselt, M. A., \& D'haenen, H. (2008). Left and right high frequency repetitive transcranial magnetic stimulation of the dorsolateral prefrontal cortex does not affect mood in female volunteers. Clinical Neurophysiology, 119(3), 568-575. doi:10.1016/j. clinph.2007.11.044

Beck, A. T., Steer, R. A., \& Brown, G. K. (1996). Manual for the beck depression inventory - II. San Antonio: Psychological Corporation.

Blackhart, G. C., Minnix, J. A., \& Kline, J. P. (2006). Can EEG asymmetry patterns predict future development of anxiety and depression? Biological Psychology, 72(1), 46-50. doi:10.1016/j. biopsycho.2005.06.010

Brunoni, A. R., Amadera, J., Berbel, B., Volz, M. S., Rizzerio, B. G., \& Fregni, F. (2011). A systematic review on reporting and assessment of adverse effects associated with transcranial direct current stimulation. The International Journal of Neuropsychopharmacology, 14(8), 1133-1145. doi:10.1017/S1461145710001690

Clarke, P. J. F., Browning, M., Hammond, G., Notebaert, L., \& MacLeod, C. (2014). The causal role of the dorsolateral prefrontal cortex in the modification of attentional bias: Evidence from transcranial direct current stimulation. Biological Psychiatry, 76(12), 946-952. doi:10.1016/j.biopsych.2014.03.003

Corbetta, M., \& Schulman, G. L. (2002). Control of goal-directed and stimulus-driven attention in the brain. Nature Reviews Neuroscience, 3, 201-215. doi:10.1038/nrn755

Crane, H. D., \& Steele, C. M. (1985). Generation-V dual-Purkinje-image eyetracker. Applied Optics, 24, 527-537.

Davidson, R. J. (1992). Anterior cerebral asymmetry and the nature of emotion. Brain and Cognition, 20, 125-151. doi:10.1016/02782626(92)90065-T

Davidson, R. J., Ekman, P., Saron, C. D., Senulis, J. A., \& Friesen, W. V. (1990). Approach-withdrawal and cerebral asymmetry: Emotional expression and brain physiology: I. Journal of Personality and Social Psychology, 58(2), 330-341. doi:10.1037/00223514.58.2.330

De Raedt, R., \& Koster, E. H. W. (2010). Understanding vulnerability for depression from a cognitive neuroscience perspective: A reappraisal of attentional factors and a new conceptual framework. Cognitive, Affective, \& Behavioral Neuroscience, 10(1), 50-70. doi:10.3758 /CABN.10.1.50
De Raedt, R., Leyman, L., Baeken, C., Van Schuerbeek, P., Luypaert, R., Vanderhasselt, M. A., \& Dannlowski, U. (2010). Neurocognitive effects of HF-rTMS over the dorsolateral prefrontal cortex on the attentional processing of emotional information in healthy women: An event-related fMRI study. Biological Psychology, 85(3), 487495. doi:10.1016/j.biopsycho.2010.09.015

De Raedt, R., Vanderhasselt, M. A., \& Baeken, C. (2015). Neurostimulation as an intervention for treatment resistant depression: From research on mechanisms towards targeted neurocognitive strategies. Clinical Psychology Review, 41, 61-69. doi:10.1016/j.cpr.2014.10.006

Debener, S., Beauducel, A., Nessler, D., Brocke, B., Heilemann, H., \& Kayser, J. (2000). Is resting anterior EEG alpha asymmetry a trait marker for depression? Findings for healthy adults and clinically depressed patients. Neuropsychobiology, 41(1), 31-37. doi: $10.1159 / 000026630$

Disner, S. G., Beevers, C. G., Haigh, E. A., \& Beck, A. T. (2011). Neural mechanisms of the cognitive model of depression. Nature Reviews Neuroscience, 12(8), 467-477. doi:10.1038/nrn3027

Ellenbogen, M. A., Schwartzman, A. E., Stewart, J., \& Walker, C. D. (2002). Stress and selective attention: The interplay of mood, cortisol levels, and emotional information processing. Psychophysiology, 39(6), 723-732. doi:10.1017/S0048577202010739

Everaert, J., Duyck, W., \& Koster, E. H. W. (2014). Attention, interpretation, and memory biases in subclinical depression: A proof-ofprinciple test of the combined cognitive biases hypothesis. Emotion, 14(2), 331-340. doi:10.1037/a0035250

Fragopanagos, N., Kockelkoren, S., \& Taylor, J. G. (2005). A neurodynamic model of the attentional blink. Cognitive Brain Research, 24(3), 568-586. doi:10.1016/j.cogbrainres.2005.03.010

Giglia, G., Brighina, F., Rizzo, S., Puma, A., Indovino, S., Maccora, S., ... Fierro, B. (2014). Anodal transcranial direct current stimulation of right dorsolateral prefrontal cortex enhances memory-guided responses in a visuospatial working memory task. Functional Neurology, 29(3), 189-193. doi:10.11138/FNeur/2014.29.3.189

Gotlib, I. H. (1998). EEG alpha asymmetry, depression, and cognitive functioning. Cognition \& Emotion, 12(3), 449-478. doi:10.1080 /026999398379673

Gross, J. J., Sheppes, G., \& Urry, H. L. (2011). Cognition and emotion lecture at the 2010 SPSP emotion preconference. Cognition \& Emotion, 25(5), 765-781. doi:10.1080/02699931.2011.555753

Heeren, A., Baeken, C., Vanderhasselt, M. A., Philippot, P., \& De Raedt, R. (2015). Impact of anodal and cathodal transcranial direct current stimulation over the left dorsolateral prefrontal cortex during attention bias modification: An eye-tracking study. PLOS ONE, 10(4), e0124182. doi:10.1371/journal.pone.0124182

Heeren, A., De Raedt, R., Koster, E. H. W., \& Philippot, P. (2013). The (neuro)cognitive mechanisms behind attention bias modification in anxiety: Proposals based on theoretical accounts of attentional bias. Frontiers in Human Neuroscience, 7, 119. doi:10.3389 /fnhum.2013.00119

Heinze, K., Ruh, N., Nitschke, K., Reis, J., Fritsch, B., Unterrainer, J. M., ... Kaller, C.P. (2014). Transcranial direct current stimulation over left and right DLPFC: Lateralized effects on planning performance and related eye movements. Biological Psychology, 102, 130-140. doi:10.1016/j.biopsycho.2014.07.019

Johnstone, T., van Reekum, C. M., Urry, H. L., Kalin, N. H., \& Davidson, R. J. (2007). Failure to regulate: Counterproductive recruitment of top-down prefrontal-subcortical circuitry in major depression. The Journal of Neuroscience: The Official Journal of the Society for Neuroscience, 27(33), 8877-8884. doi:10.1523/JNEUROSCI.206307.2007

Joormann, J. (2004). Attentional bias in dysphoria: The role of inhibitory processes. Cognition \& Emotion, 18(1), 125-147. doi:10.1080 /02699930244000480 
Joormann, J., \& D’Avanzato, C. (2010). Emotion regulation in depression: Examining the role of cognitive processes. Cognition \& Emotion, 24(6), 913-939. doi:10.1080/02699931003784939

Joormann, J., Yoon, K. L., \& Zetsche, U. (2007). Cognitive inhibition in depression. Applied and Preventive Psychology, 12(3), 128-139. doi:10.1016/j.appsy.2007.09.002

Keeser,D., Meindl, T., Bor, J., Palm, U., Pogarell, O., Mulert, C., ... Padberg, F. (2011). Prefrontal transcranial direct current stimulation changes connectivity of resting-state networks during fMRI. The Journal of Neuroscience, 31(43), 15284-15293. doi:10.1523 /JNEUROSCI.0542-11.2011

Kluger, B. M., \& Triggs, W. J. (2007). Use of transcranial magnetic stimulation to influence behavior. Current Neurology and Neuroscience Reports, 7(6), 491-497. doi:10.1007/s11910-0070076-5

Koole, S. L. (2009). The psychology of emotion regulation: An integrative review. Cognition \& Emotion, 23(1), 4-41. doi:10.1080 /02699930802619031

Langner, O., Dotsch, R., Bijlstra, G., Wigboldus, D. H. J., Hawk, S. T., \& van Knippenberg, A. (2010). Presentation and validation of the radboud faces database. Cognition \& Emotion, 24(8), 1377-1388. doi:10.1080/02699930903485076

Leyman, L., De Raedt, R., Vanderhasselt, M. A., \& Baeken, C. (2009). Influence of high-frequency repetitive transcranial magnetic stimulation over the dorsolateral prefrontal cortex on the inhibition of emotional information in healthy volunteers. Psychological Medicine, 39, 1019-1028. doi:10.1017/S0033291708004431

Liotti, M., \& Mayberg, H. S. (2001). The role of functional neuroimaging in the neuropsychology of depression. Journal of Clinical and Experimental Neuropsychology, 23(1), 121-136. doi:10.1076 /jcen.23.1.121.1223

McCormack, H. M., Horne, D. J., \& Sheather, S. (1988). Clinical applications of visual analogue scales: a critical review. Psychological Medicine, 18, 1007-1019.

MacDonald, A. W., Cohen, J. D., Stenger, V. A., \& Carter, C. S. (2000). Dissociating the role of the dorsolateral prefrontal and anterior cingulate cortex in cognitive control. Science, 288, 1835-1838. doi:10.1126/science. 288.5472 .1835

Morris, J. S., Friston, K. J., Büchel, C., Frith, C. D., Young, A. W., Calder, A. J., \& Dolan, R. J. (1998). A neuromodulatory role for the human amygdala in processing emotional facial expressions. Brain, 121, 47-57. doi:10.1093/brain/121.1.47

Nitsche, M.A., Cohen, L., Wassermann, E.M., Priori, A., Lang, N., Antal, A., ... Pascual-Leone, A. (2008). Transcranial direct current stimulation: State of the art 2008. Brain Stimulation, 1(3), 206-223. doi:10.1016/j.brs.2008.06.004

Nitsche, M.A., Fricke, K., Henschke, U., Schlitterlau, A., Liebetanz, D., Lang, N., ... Paulus, W. (2003). Pharmacological modulation of cortical excitability shifts induced by transcranial direct current stimulation in humans. The Journal of Physiology, 553(1), 293-301. doi:10.1113/jphysiol.2003.049916

Nitsche, M. A., Koschack, J., Pohlers, H., Hullemann, S., Paulus, W., \& Happe, S. (2012). Effects of frontal transcranial direct current stimulation on emotional state and processing in healthy humans. Frontiers in Psychiatry, 3(June), 1-10. doi:10.3389 /fpsyt.2012.00058

Nitsche, M. A., \& Paulus, W. (2000). Excitability changes induced in the human motor cortex by weak transcranial direct current stimulation. Journal of Physiology, 527(3), 633-639. doi:10.1111/j.14697793.2000.t01-1-00633.x

Nitsche, M. A., \& Paulus, W. (2001). Sustained excitability elevations induced by transcranial DC motor cortex stimulation in humans. Neurology, 57(10), 1899-1901.

Nitschke, J. B., \& Heller, W. (2005). Distinguishing neural substrates of heterogeneity among anxiety disorders. International Review of Neurobiology, 67(05), 1-42. doi:10.1016/S0074-7742(05)67001-8
Ochsner, K. N., \& Gross, J. J. (2005). The cognitive control of emotion. Trends in Cognitive Sciences, 9(5), 242-249. doi:10.1016/j. tics.2005.03.010

Overbeek, T., Schruers, K., \& Griez, E. (1999). Mini International Neuropsychiatric Interview: Nederlandse Versie 5.0.0. Maastricht: Universiteit van Maastricht.

Posner, M. I., \& Cohen, Y. (1984). Components of visual orienting. In H. Bouma \& D. Bouwhuis (Eds.), Attention and performance (Vol. X, pp. 531-556). Hove: Erlbaum.

Remue, J., Baeken, C., \& De Raedt, R. (2016). Does a single neurostimulation session really affect mood in healthy individuals? A systematic review. Neuropsychologia, 85, 184-198. doi:10.1016 /j.neuropsychologia.2016.03.012

Rounis, E., Stephan, K. E., Lee, L., Siebner, H. R., Pesenti, A., Friston, K. J., ... Frackowiak, R. S. (2006). Acute changes in frontoparietal activity after repetitive transcranial magnetic stimulation over the dorsolateral prefrontal cortex in a cued reaction time task. The Journal of Neuroscience, 26(38), 9629-9638. doi:10.1523 /JNEUROSCI.2657-06.2006

Sanchez, A., Everaert, J., De Putter, L., Mueller, S., \& Koster, E. H. W. (2015). Life is...great ! Emotional attention during instructed and uninstructed ambiguity resolution in relation to depressive symptoms. Biological Psychology, 109, 67-72. doi:10.1016/j. biopsycho.2015.04.007

Sanchez, A., Vazquez, C., Gomez, D., \& Joormann, J. (2014). Gazefixation to happy faces predicts mood repair after a negative mood induction. Emotion, 14(1), 85-94. doi:10.1037/a0034500

Sanchez, A., Vazquez, C., Marker, C., LeMoult, J., \& Joormann, J. (2013). Attentional disengagement predicts stress recovery in depression: An eye-tracking study. Journal of Abnormal Psychology, 122(2), 303-313. doi:10.1037/a0031529

Segrave, R. A., Arnold, S., Hoy, K., \& Fitzgerald, P. B. (2013). Concurrent cognitive control training augments the antidepressant efficacy of tDCS: a pilot study. Brain Stimulation, 7(2), 325-331. doi:10.1016/j.brs.2013.12.008

Seminowicz, D., Mayberg, H., McIntosh, A., Goldapple, K., Kennedy, S., Segal, Z., \& Rafi-Tari, S. (2004). Limbic-frontal circuitry in major depression: A path modeling metanalysis. NeuroImage, 22(1), 409-418. doi:10.1016/j.neuroimage.2004.01.015

Sheehan, D. V., Lecrubier, Y., Sheehan, K. H., Amorim, P., Janavs, J., Weiller, E., ... Dunbar, G. C. (1998). The Mini-International Neuropsychiatric Interview (M.I.N.I.): the development and validation of a structured diagnostic psychiatric interview for DSM-IV and ICD-10. The Journal of Clinical Psychiatry, 59(Suppl 20), 22-33.

Spielberger, C. D., Gorsuch, R. L., Lushene, R., Vagg, P. R., \& Jacobs, G. A. (1983). Manual for the state-trait anxiety inventory. Palo Alto: Consulting Psychologists Press.

Stagg, C.J., Best, J.G., Stephenson, N.C., O’Shea1, J., Wylezinska, M., Kincses, Z. T., ... Johansen-Berg, H. (2013). Polarity-sensitive modulation of cortical neurotransmitters by transcranial stimulation. The Journal of Neuroscience, 29(16), 5202-5206. doi:10.1523 /JNEUROSCI.4432-08.2009

Van der Does, A. J. W. (2002). Handleiding bij de Nederlandse versie van de Beck Depression Inventory -second edition (BDI-II-NL). [The Dutch version of the Beck Depresion Inventory-II]. Lisse: Swets \& Zeitlinger.

van der Ploeg, H. M., Defares, P. B., \& Spielberger, C. D. (1980). Handleiding bij de ZelfBeoordelings Vragenlijst, ZBV. Een Nederlandstalige bewerking van de Spielberger StateTrait Anxiety Inventory, STATDY. Lisse: Swets en Zeitlinger.

Vanderhasselt, M. A., Brunoni, A. R., Loeys, T., Boggio, P. S., \& De Raedt, R. (2013). Nosce te ipsum-Socrates revisited? Controlling momentary ruminative self-referent thoughts by neuromodulation of emotional working memory. Neuropsychologia, 51, 2581-2589. doi:10.1016/j.neuropsychologia.2013.08.011 
Vanderhasselt, M. A., De Raedt, R., Baeken, C., Leyman, L., \& D'haenen, H. (2006). The influence of rTMS over the left dorsolateral prefrontal cortex on Stroop task performance. Experimental Brain Research, 169(2), 279-282. doi:10.1007 /s00221-005-0344-Z

Vanderhasselt, M. A., De Raedt, R., Brunoni, A. R., Campanhã, C., Baeken, C., Remue, J., \& Boggio, P. S. (2013). tDCS over the left prefrontal cortex enhances cognitive control for positive affective stimuli. PLoS ONE, 8(5), 4-9. doi:10.1371/journal.pone.0062219
Vanderhasselt, M. A., Remue, J., Ng, K. K., \& De Raedt, R. (2014). The interplay between the anticipation and subsequent online processing of emotional stimuli as measured by pupillary dilatation: The role of cognitive reappraisal. Frontiers in Psychology, 5, 1-11. doi:10.3389 /fpsyg.2014.00207

Wager, T. D., Davidson, M. L., Hughes, B. L., Lindquist, M. A., \& Ochsner, K. N. (2008). Prefrontal-subcortical pathways mediating successful emotion regulation. Neuron, 59(6), 1037-1050. doi:10.1016/j. neuron.2008.09.006 